\title{
MHD Mixed Convective Boundary Layer Flow of a Nanofluid through a Porous Medium due to an Exponentially Stretching Sheet
}

\author{
M. Ferdows, ${ }^{1}$ Md. Shakhaoath Khan, ${ }^{2}$ \\ Md. Mahmud Alam, ${ }^{2}$ and Shuyu Sun ${ }^{3}$ \\ ${ }^{1}$ Department of Mathematics, University of Dhaka, Dhaka 1000, Bangladesh \\ ${ }^{2}$ Science, Engineering and Technology School, Khulna University, Khulna 9208, Bangladesh \\ ${ }^{3}$ Division of Applied Mathematics and Computational Science, King Abdullah University of Science and \\ Technology, Thuwal, Saudi Arabia \\ Correspondence should be addressed to M. Ferdows, ferdows@du.ac.bd
}

Received 22 May 2012; Revised 29 July 2012; Accepted 13 August 2012

Academic Editor: Ming Li

Copyright (C) 2012 M. Ferdows et al. This is an open access article distributed under the Creative Commons Attribution License, which permits unrestricted use, distribution, and reproduction in any medium, provided the original work is properly cited.

\begin{abstract}
Magnetohydrodynamic (MHD) boundary layer flow of a nanofluid over an exponentially stretching sheet was studied. The governing boundary layer equations are reduced into ordinary differential equations by a similarity transformation. The transformed equations are solved numerically using the Nactsheim-Swigert shooting technique together with Runge-Kutta sixorder iteration schemes. The effects of the governing parameters on the flow field and heat transfer characteristics were obtained and discussed. The numerical solutions for the wall skin friction coefficient, the heat and mass transfer coefficient, and the velocity, temperature, and concentration profiles are computed, analyzed, and discussed graphically. Comparison with previously published work is performed and excellent agreement is observed.
\end{abstract}

\section{Introduction}

The study of Magnetohydrodynamics (MHD) boundary layer flow on a continuous stretching sheet has attracted considerable attention during the last few decades due to its numerous applications in industrial manufacturing processes such as the aerodynamic extrusion of plastic sheets, liquid film, hot rolling, wire drawing, glass fiber and paper production, drawing of plastic films, metal, and polymer extrusion, and metal spinning. Crane [1] was the first to consider the boundary layer flow caused by a stretching sheet which moves with a velocity varying linearly with the distance from a fixed point. The heat transfer aspect of this problem was investigated by Carragher and Crane [2] under the conditions when the temperature difference between the surface and the ambient fluid is proportional 
to a power of the distance from a fixed point. After this pioneering work, the flow field over an exponentially stretching surface has drawn considerable attention and a good amount of literature has been generated on this problem. Magyari and Keller [3] analyzed the steady boundary layers on an exponentially stretching continuous surface with an exponential temperature distribution. Partha et al. [4] investigated the effect of viscous dissipation on the mixed convection heat transfer from an exponentially stretching surface. Sajid and Hayat [5] studied the influence of thermal radiation on the boundary layer flow due to an exponentially stretching sheet by solving the problem analytically via homotopy analysis method (HAM). The study of magnetohydrodynamic (MHD) has important applications, and may be used to deal with problems such as cooling of nuclear reactors by liquid sodium and induction flow meter, which depends on the potential difference in the fluid in the direction perpendicular to the motion and to the magnetic field [6]. At high operating temperature, radiation effect can be quite significant. Many processes in engineering areas occur at high temperatures and knowledge of radiation heat transfer becomes very important for the design of pertinent equipment [7]. Elbashbeshy [8] added new dimension to the study on exponentially continuous stretching surface. Khan [9] and Sanjayanand and Khan [10] studied the viscous-elastic boundary layer flow and heat transfer due to an exponentially stretching sheet. The numerical simulation of boundary layer flow over an exponentially stretching sheet with thermal radiation was given by Bidin and Nazar [11]. Wang [12] studied the free convection on a vertical stretching surface, also Reddy Gorla and Sidawi [13] investigated the free convection on a vertical stretching surface with suction and blowing.

Dissipation is the process of converting mechanical energy of downward-flowing water into thermal and acoustical energy. Vajravelu and Hadjinicolaou [14] analyzed the heat transfer characteristics over a stretching surface with viscous dissipation in the presence of internal heat generation or absorption. Convective boundary layer flow has wide applications in engineering as postaccidental heat removal in nuclear reactors, solar collectors, drying processes, heat exchangers, geothermal and oil recovery, building construction, and so forth. Cheng and Minkowycz [15] also studied free convection from a vertical flat plate with applications to heat transfer from a dick. Gorla et al. [16, 17] solved the nonsimilar problem of free convective heat transfer from a vertical plate embedded in a saturated porous medium with an arbitrary varying surface temperature.

The term nanofluid refers to these kinds of fluids by suspending nanometer sized metallic particles in common fluids and reported that their highly enhanced thermal properties has been introduced by Choi [18]. Kang et al. [19] investigated the estimation of thermal conductivity of nanofluid using experimental effective particle volume. Abu-Nada et al. $[20,21]$ have showed application of nanofluids for heat transfer and also analyzed effects of inclination angle on natural convection in enclosures filled with $\mathrm{Cu}$-water nanofluid. Wang and Mujumdar $[22,23]$ studied heat transfer characteristics of nanofluids including theoretical and numerical investigations. Ghasemi and Aminossa dati [24] have showed the periodic natural convection in a nanofluid filled enclosure with oscillating heat flux.

The study of convective instability and heat transfer characteristics of the nanofluids was considered by Kim et al. [25]. Jang and Choi [26] obtained nanofluids thermal conductivity and various parameters effect this study. The natural convective boundary layer flows of a nanofluid past a vertical plate have been described by Neild and Kuznestov [27] and Kuznestov and Neild [28]. In this model, Brownian motion and Thermophoresis are accounted with the simplest possible boundary conditions. They also studied ChengMinkowycz problem for natural convective boundary-layer flow in a porous medium saturated by a nanofluid. Owing to applications in science and engineering, Brownian motion 


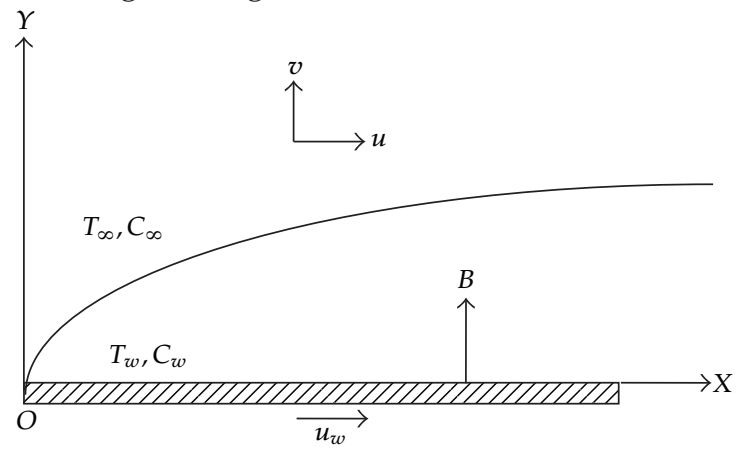

Figure 1: Physical model and coordinate system.

was reported by [29-36]. Bachok et al. [37] have showed the steady boundary layer flow of a nanofluid past a moving semiinfinite flat plate in a uniform free stream. It was assumed that the plate is moving in the same or opposite directions to the free stream to define resulting system of nonlinear ordinary differential equations.

Khan and Pop [38, 39] formulated the problem of laminar boundary layer flow of a nanofluid past a stretching sheet. They also expressed free convection boundary layer nanofluid flow past a horizontal flat plate. Hamad and Pop [40] discussed the boundary layer flow near the stagnation-point flow on a permeable stretching sheet in a porous medium saturated with a nanofluid. Hamad et al. [41] investigates free convection flow of a nanofluid past a semiinfinite vertical flat plate with the influence of magnetic field. Very recently Shakhaoath et al. $[42,43]$ investigate the effects of thermal radiation and magnetic field on the boundary layer flow of a nanofluid over a stretching surface.

The present paper studies the problem of MHD mixed convective boundary layer of a nanofluid flow over an exponentially stretching sheet. The governing equations are transformed into nonlinear coupled ordinary differential equations which depends on the combined porous and magnetic parameter $(R)$, thermal convective parameter $\left(\lambda_{T}\right)$, Mass convective parameter $\left(\lambda_{M}\right)$, viscosity ratio parameter $(\Lambda)$, dimensionless inertia parameter $(\nabla)$, Prandtl number $\left(P_{r}\right)$, Eckert number $\left(E_{c}\right)$, Lewis number $\left(L_{e}\right)$, Brownian motion parameter $\left(N_{b}\right)$, and Thermophoresis parameter $\left(N_{t}\right)$. The obtained nonlinear coupled ordinary differential equations are solved numerically using Nactsheim-Swigert [44] shooting iteration technique together with Runge-Kutta six-order iteration schemes. The velocity, temperature, and concentration distributions are discussed and presented graphically, and also the skin-friction coefficient, the surface heat, and mass transfer rate at the sheet are investigated.

\section{Mathematical Formulation}

Consider a steady two-dimensional flow of an incompressible viscous and electrically conducting nanofluid caused by a stretching sheet, which is placed in a quiescent ambient fluid of uniform temperature of the plate and species concentration are raised to $T_{w}\left(>T_{\infty}\right)$ and $C_{w}\left(>C_{\infty}\right)$, respectively, which are thereafter maintained constant, where $T_{w}, C_{w}$ are temperature and species concentration at the wall and $T_{\infty}, C_{\infty}$ are temperature and species concentration far away from the plate, respectively. The $x$-axis is taken along the stretching sheet in the direction of the motion and $y$-axis is perpendicular to it. Consider that a variable magnetic field $B(x)$ is applied normal to the sheet and that the induced magnetic field is neglected, which is justified for MHD flow at small magnetic Reynolds number. The sketch of the physical configuration and coordinate system are shown in Figure 1. 
Under the above assumptions and usual boundary layer approximation, the MHD free convective nanofluid flow and heat and mass transfer are governed by the following (see Nield and Kuznetsov [27] and Kuznetsov and Nield [28]):

$$
\begin{gathered}
\frac{\partial u}{\partial x}+\frac{\partial v}{\partial y}=0 \\
u \frac{\partial u}{\partial x}+v \frac{\partial u}{\partial y}=\widetilde{v} \frac{\partial^{2} u}{\partial y^{2}}-\frac{v}{K} u-c^{*} \varepsilon^{2} u^{2}-\frac{\sigma B^{2}}{\rho} u+g \beta\left(T-T_{\infty}\right)+g \beta^{*}\left(C-C_{\infty}\right) \\
u \frac{\partial T}{\partial x}+v \frac{\partial T}{\partial y}=\alpha \frac{\partial^{2} T}{\partial y^{2}}+\frac{\tilde{v}}{c_{p}}\left(\frac{\partial u}{\partial y}\right)^{2}+\tau\left\{D_{B}\left(\frac{\partial T}{\partial y} \cdot \frac{\partial C}{\partial y}\right)+\frac{D_{T}}{T_{\infty}}\left(\frac{\partial T}{\partial y}\right)^{2}\right\} \\
u \frac{\partial C}{\partial x}+v \frac{\partial C}{\partial y}=D_{B} \frac{\partial^{2} C}{\partial y^{2}}+\frac{D_{T}}{T_{\infty}} \frac{\partial^{2} T}{\partial y^{2}}
\end{gathered}
$$

where $u$ and $v$ are the velocities in the $x$ - and $y$-directions, respectively, $\rho$ is the fluid density, $v$ the kinematic viscosity, $\widetilde{v}$ the reference kinematic viscosity, $K$ the variable thermal conductivity, $c_{p}$ the specific heat at constant pressure, $T$ and $C$ the fluid temperature and concentration in the boundary layer, $c^{*} \varepsilon^{2}$ is the inertia parameter, $\alpha$ is the thermal diffusivity, $D_{B}$ is the Brownian diffusion coefficient, and $D_{T}$ is the thermophoresis diffusion coefficient.

The boundary condition for the model is

$$
\begin{aligned}
& u=u_{w}=u_{\circ} e^{x / L}, \quad v=0, \quad T=T_{w}=T_{\infty}+T_{\circ} e^{x / 2 L}, \\
& C=C_{w}=C_{\infty}+C_{\circ} e^{x / 2 L}, \quad \text { at } y=0 \\
& u=0, \quad T \longrightarrow T_{\infty}, \quad C \longrightarrow C_{\infty}, \quad \text { as } y \longrightarrow \infty,
\end{aligned}
$$

where $u_{\circ}$ is the reference velocity, $T_{\circ}, C_{\circ}$ the reference temperature and concentration, respectively, and $L$ is the reference length. To obtain similarity solutions, it is assumed that the magnetic field $B(x)$ is of the form

$$
B=B_{\circ} e^{x / 2 L}
$$

where $B_{\circ}$ is the constant magnetic field. Also the variable thermal conductivity can be taken as the form

$$
K=k_{\circ} e^{x / 2 L}
$$


In order to attains a similarity solution to (2) with the boundary conditions (2.2), the following dimensionless variables are used:

$$
\begin{gathered}
\eta=y \sqrt{\frac{u_{\circ}}{2 v L}} e^{x / 2 L}, \quad \psi=\sqrt{2 v u_{\circ} L} e^{x / 2 L} f(\eta), \\
\theta=\theta(\eta)=\frac{T-T_{\infty}}{T_{w}-T_{\infty}}, \quad \varphi=\varphi(\eta)=\frac{C-C_{\infty}}{C_{w}-C_{\infty}}, \\
u=\frac{\partial \psi}{\partial y}=u_{\circ} e^{x / L} f^{\prime}, \quad v=-\frac{\partial \psi}{\partial x}=-\sqrt{\frac{v u_{\circ}}{2 L}} e^{x / 2 L}\left(f+\eta f^{\prime}\right) .
\end{gathered}
$$

From the above transformations, the nondimensional, nonlinear, and coupled ordinary differential equations are obtained as

$$
\begin{gathered}
\wedge f^{\prime \prime \prime}+f f^{\prime \prime}-2(\nabla+1) f^{\prime 2}-2 R f^{\prime}+2 \lambda_{T} \theta+2 \lambda_{M} \varphi=0 \\
\theta^{\prime \prime}-P_{r} f^{\prime} \theta+P_{r} f \theta^{\prime}+\wedge P_{r} E_{c} f^{\prime \prime 2}+P_{r} N_{b} \theta^{\prime} \varphi^{\prime}+P_{r} N_{t} \theta^{\prime 2}=0 \\
\varphi^{\prime \prime}+L_{e} f \varphi^{\prime}-L_{e} f^{\prime} \varphi+\left(\frac{N_{t}}{N_{b}}\right) \theta^{\prime \prime}=0,
\end{gathered}
$$

where the notation primes denote differentiation with respect to $\eta$ and the parameters are defined as

$$
\begin{gathered}
R=\left(\frac{v L}{k_{\circ} u_{\circ}}+\frac{\sigma B_{\circ}^{2} L}{\rho u_{\circ}}\right) \quad(\text { combined porous and magnetic parameter), } \\
\wedge=\frac{\widetilde{v}}{v} \quad \text { (viscosity ratio parameter), } \\
\nabla=c^{*} \varepsilon^{2} L \quad(\text { dimensionless inertia parameter), } \\
\lambda_{T}=\frac{G_{r}}{R_{e_{x}}^{2}}=\frac{g \beta\left(T_{w}-T_{\infty}\right)}{u_{w}^{2}} \quad \text { (thermal convective parameter), } \\
\lambda_{M}=\frac{G_{m}}{R_{e_{x}}^{2}}=\frac{g \beta\left(C_{w}-C_{\infty}\right)}{u_{w}^{2}} \quad \text { (mass convective parameter), } \\
p_{r}=\frac{v}{\alpha} \quad(\text { Prandtl number), } \\
E_{c}=\frac{u_{\circ}^{2}}{T_{\circ} c_{p}} \quad \text { (Eckert number), } \\
N_{B}=\frac{(\rho c)_{p} D_{B}\left(C_{w}-C_{\infty}\right)}{v(\rho c)_{f}} \quad \text { (Brownian motion parameter), }
\end{gathered}
$$




$$
\begin{gathered}
N_{t}=\frac{(\rho c)_{p} D_{T}\left(T_{w}-T_{\infty}\right)}{v T_{\infty}(\rho c)_{f}} \quad \text { (Thermophoresis parameter), } \\
L_{e}=\frac{v}{D_{B}} \quad \text { (Lewis number). }
\end{gathered}
$$

The transformed boundary conditions

$$
\begin{gathered}
f=0, \quad f^{\prime}=1, \quad \theta=1, \quad \varphi=1, \quad \text { at } \eta=0 \\
f^{\prime}=0, \quad \theta=0, \quad \varphi=0, \quad \text { as } \eta \longrightarrow \infty .
\end{gathered}
$$

The physical quantities of the skin-friction coefficient, the reduced Nusselt number, and reduced Sherwood number are calculated, respectively, by the following:

$$
C_{f}\left(R_{e_{x}}\right)^{-1 / 2}=-f^{\prime \prime}(0), \quad N_{u}\left(R_{e_{x}}\right)^{-1 / 2}=-\theta^{\prime}(0), \quad S_{h}\left(R_{e_{x}}\right)^{-1 / 2}=-\varphi^{\prime}(0),
$$

where $R_{-}\left(e_{-} x\right)=\left(x u_{\circ} e^{x / L}\right) / v$ is the local Reynolds number.

\section{Numerical Technique}

The system of nondimensional, nonlinear, and coupled ordinary differential equations (2.6) with boundary condition (2.8) are solved numerically using standard initially value solver the shooting method. For the purpose of this method, the Nactsheim-Swigert shooting iteration technique [44] together with Runge-Kutta six order iteration scheme is taken and determines the temperature and concentration as a function of the coordinate $\eta$. Extension of the iteration shell to above equation system of differential (2.8) is straightforward; there are three asymptotic boundary condition and hence three unknown surface conditions $f^{\prime \prime}(0), \theta^{\prime}(0)$ and $\varphi^{\prime}(0)$.

\section{Results and Discussions}

The system of governing equation (2.6) are coupled both in heat and mass transfer; it is clear that analytical solution is not possible. Thus, computations have been carried out based on numerical technique. The values of the governing parameters are chosen arbitrary. However, the numerical results are presented for some representative values of these governing parameters. In order to see the physical insight, the numerical values of velocity $\left(f^{\prime}\right)$, temperature $(\theta)$, and concentration $(\varphi)$ with the boundary layer have been computed for different parameters as the combined porous and magnetic parameter $(R)$, Thermal convective parameter $\left(\lambda_{T}\right)$, Mass convective parameter $\left(\lambda_{M}\right)$, viscosity ratio parameter $(\wedge)$, dimensionless inertia parameter $(\nabla)$, Prandtl number $\left(P_{r}\right)$, Eckert number $\left(E_{c}\right)$, Lewis number $\left(L_{e}\right)$, Brownian motion parameter $\left(N_{b}\right)$, and Thermophoresis parameter $\left(N_{t}\right)$. In order to assess the accuracy of the numerical results as the results for the reduced Nusselt number $-\theta^{\prime}(0)$ for different values of Prandtl number $\left(P_{r}\right)$ and Eckert number $\left(E_{c}\right)$, the present results compared with Bidin and Nazar [11]. Comparison with the existing results 
Table 1: Comparison for the reduced Nusselt number $-\theta^{\prime}(0)$ for the values of $\lambda_{T}=\lambda_{M}=R=\nabla=L_{e}=$ $N_{b}=N_{t}=0.0$.

\begin{tabular}{ccccccc}
\hline & \multicolumn{2}{c}{$E_{c}=0.0$} & \multicolumn{2}{c}{$E_{c}=0.0$} & \multicolumn{2}{c}{$E_{c}=0.0$} \\
$P_{r}$ & $\begin{array}{c}\text { Bidin and } \\
\text { Nazar [1] } \\
(K=0.0)\end{array}$ & $\begin{array}{c}\text { Present } \\
\text { results }\end{array}$ & $\begin{array}{c}\text { Bidin and } \\
\text { Nazar }[11] \\
(K=0.0)\end{array}$ & $\begin{array}{c}\text { Present } \\
\text { results }\end{array}$ & $\begin{array}{c}\text { Bidin and } \\
\text { Nazar [11] } \\
(K=0.0)\end{array}$ & $\begin{array}{c}\text { Present } \\
\text { results }\end{array}$ \\
\hline 1 & 0.9547 & 0.9550 & 0.8622 & 0.8629 & 0.5385 & 0.5392 \\
2 & 1.4714 & 1.4719 & 1.3055 & 1.3062 & 0.7248 & 0.7250 \\
3 & 1.8691 & 1.8701 & 1.6882 & 1.6890 & 0.8301 & 0.8309 \\
\hline
\end{tabular}

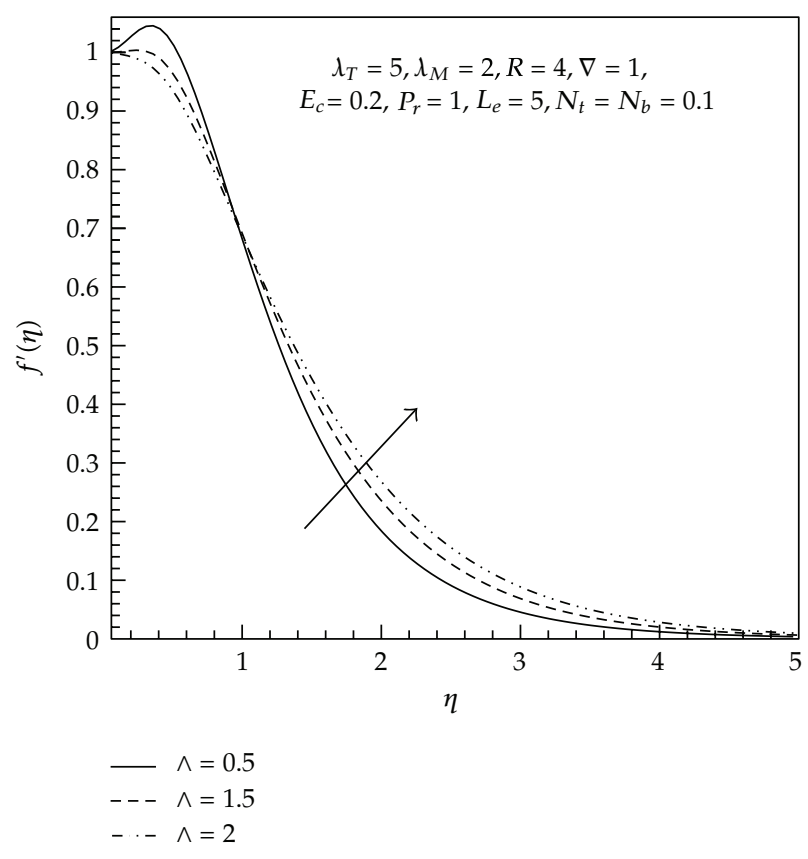

Figure 2: Effect of $\wedge$ on velocity profiles.

shows a favorable agreement, as presented in Table 1.Therefore, the present results are very accurate. The physical representation of the present study is shown in Figures 2-21.

Figure 2 displays the dimensionless velocity distribution $f^{\prime}(\eta)$ for different values of $\wedge$ where $\lambda_{T}=5.0, \lambda_{M}=2.0, R=4.0, \nabla=1.0, E_{c}=0.2, P_{r}=1.0, L_{e}=5.0, N_{t}=0.1$, and $N_{b}=$ 0.1 Then for above case it is observed that velocity profiles increase as $\wedge$ increases.

Figure 3 exhibits the dimensionless velocity distribution $f^{\prime}(\eta)$ for different values of $R$ where $\lambda_{T}=5.0, \lambda_{M}=2.0, \wedge=1.5, \nabla=1.0, E_{c}=0.2, P_{r}=1.0, L_{e}=5.0, N_{t}=0.1$, and $N_{b}=$ 0.1 . It shows that the velocity reduces steeply near the exponential stretching sheet as $R$ is increased.

Figure 4 represents the dimensionless velocity distribution $f^{\prime}(\eta)$ for different values of $\lambda_{T}$ where $\wedge=1.5, \lambda_{M}=2.0, R=4.0, \nabla=1.0, E_{c}=0.2, P_{r}=1.0, L_{e}=5.0, N_{t}=0.1$, and $N_{b}=$ 0.1 It shows that the velocity rises as $\lambda_{T}$ is increased.

Figure 5 depicts the dimensionless velocity distribution $f^{\prime}(\eta)$ for different values of $\lambda_{M}$ where $\lambda_{T}=5.0, \wedge=1.5, R=4.0, \nabla=1.0, E_{c}=0.2, P_{r}=1.0, L_{e}=5.0, N_{t}=0.1$, and $N_{b}=0.1$. It shows that the velocity reduces as $\lambda_{M}$ is increased. 


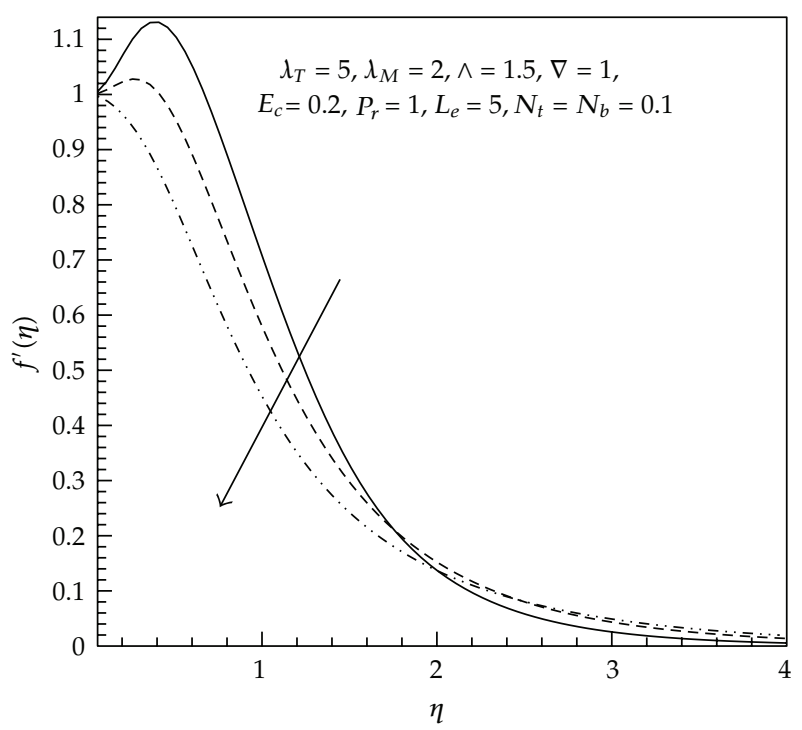

- $R=0.5$

- - $R=2.5$

- - $R=5$

Figure 3: Effect of $R$ on velocity profiles.

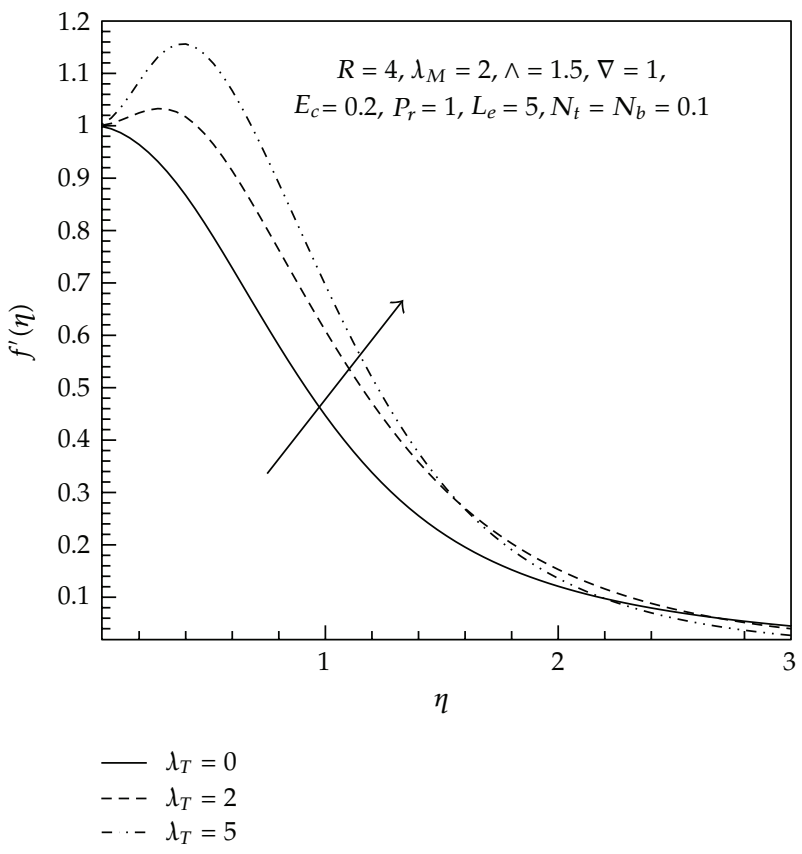

Figure 4: Effect of $\lambda_{T}$ on velocity profiles. 


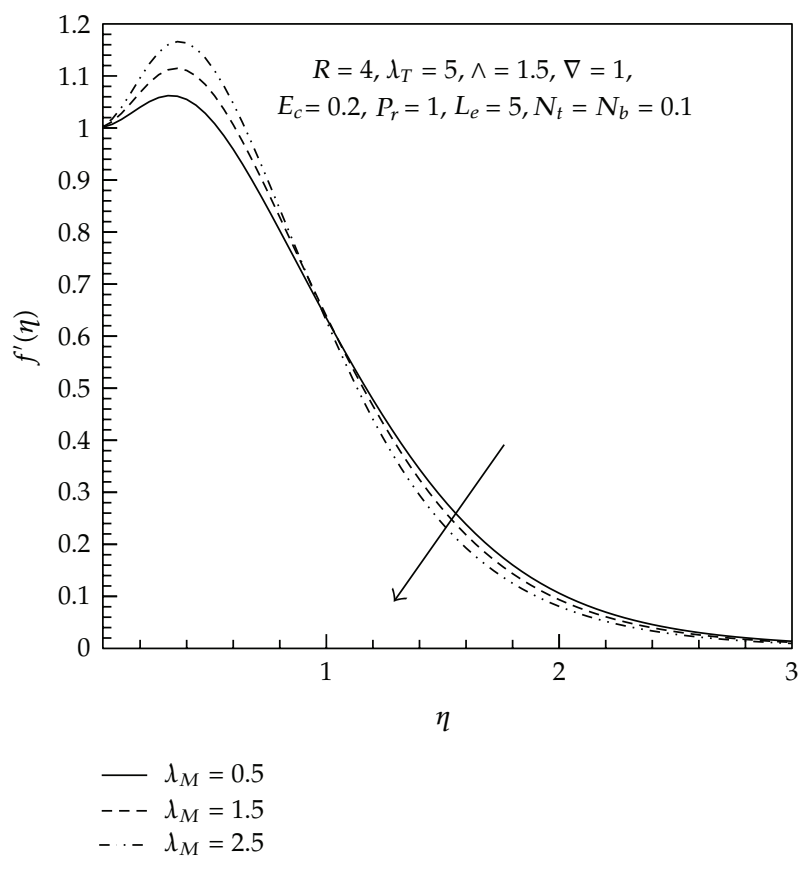

Figure 5: Effect of $\lambda_{M}$ on velocity profiles.

Figure 6 depicts the dimensionless velocity distribution $f^{\prime}(\eta)$ for different values of $E_{c}$ where $\lambda_{T}=5.0, \lambda_{M}=2.0, R=4.0, \nabla=1.0, \wedge=1.5, P_{r}=1.0, L_{e}=5.0, N_{t}=0.1$, and $N_{b}=0.1$. It shows that the velocity rises as $E_{c}$ is increased.

Figure 7 represents the dimensionless temperature distribution $\theta(\eta)$ for different values of $\wedge$ where $\lambda_{T}=5.0, \lambda_{M}=2.0, R=4.0, \nabla=1.0, E_{c}=0.2, P_{r}=1.0, L_{e}=5.0, N_{t}=$ 0.1 , and $N_{b}=0.1$. It shows that the thermal boundary layer rises as $\wedge$ is increased causing the fluid temperature to reduce at every point other than the wall.

Figure 8 shows the dimensionless temperature distribution $\theta(\eta)$ for different values of $R$ where $\lambda_{T}=5.0, \lambda_{M}=2.0, \wedge=1.5, \nabla=1.0, E_{c}=0.2, P_{r}=1.0, L_{e}=5.0, N_{t}=0.1$ and $N_{b}=$ 0.1 It shows that the thermal boundary layer increases as $R$ is increased.

Figure 9 exhibits the dimensionless temperature distribution $\theta(\eta)$ for different values of $\lambda_{T}$ where $\wedge=1.5, \lambda_{M}=2.0, R=4.0, \nabla=1.0, E_{c}=0.2, P_{r}=1.0, L_{e}=5.0, N_{t}=$ 0.1 , and $N_{b}=0.1$. It shows that the thermal boundary layer reduces as $\lambda_{T}$ is increased.

Figure 10 portrays the dimensionless temperature distribution $\theta(\eta)$ for different values of $\lambda_{M}$ where $\lambda_{T}=5.0, \wedge=1.5, R=4.0, \nabla=1.0, E_{c}=0.2, P_{r}=1.0, L_{e}=5.0, N_{t}=0.1$, and $N_{b}=$ 0.1. It shows that the thermal boundary layer rises as $\lambda_{M}$ is increased.

Figure 11 shows the dimensionless temperature distribution $\theta(\eta)$ for different values of $E_{c}$ where $\lambda_{T}=5.0, \lambda_{M}=2.0, R=4.0, \nabla=1.0, \wedge=1.5, P_{r}=1.0, L_{e}=5.0, N_{t}=0.1$, and $N_{b}=$ 0.1 . It shows that the thermal boundary layer increases gradually as $E_{c}$ is increased.

Figure 12 shows the dimensionless concentration distribution $\varphi(\eta)$ for different values of $\wedge$ where $\lambda_{T}=5.0, \lambda_{M}=2.0, R=4.0, \nabla=1.0, E_{c}=0.2, P_{r}=1.0, L_{e}=5.0, N_{t}=$ 0.1 , and $N_{b}=0.1$. It shows that the concentration boundary layer rises as $\wedge$ is increases.

Figure 13 exhibits the dimensionless concentration distribution $\varphi(\eta)$ for different values of $R$ where $\lambda_{T}=5.0, \lambda_{M}=2.0, \wedge=1.5, \nabla=1.0, E_{c}=0.2, P_{r}=1.0, L_{e}=5.0, N_{t}=$ 0.1 , and $N_{b}=0.1$. It shows that the concentration boundary layer rises as $R$ is increased. 


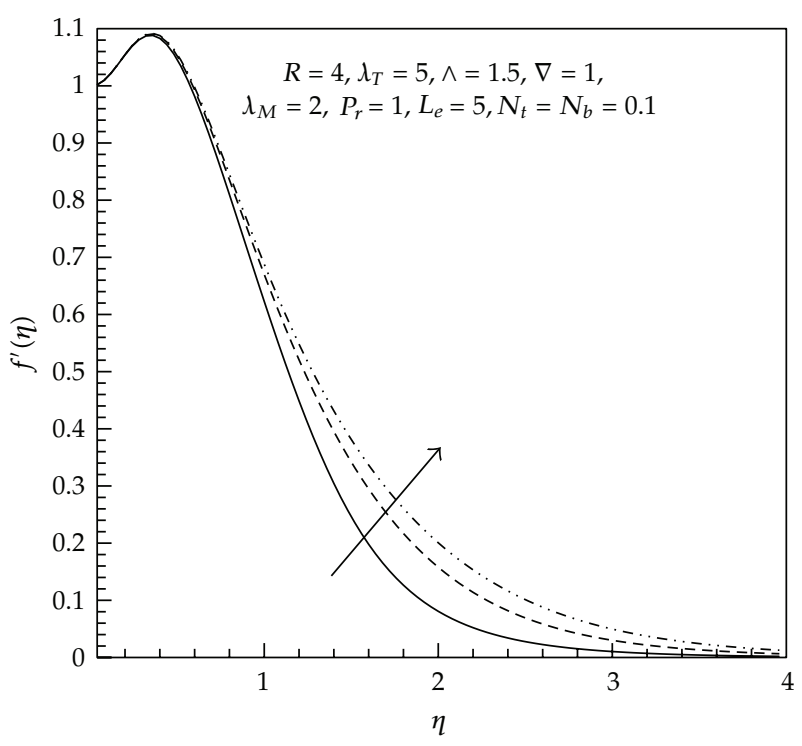

$-E_{c}=0$

- - $E_{c}=0.5$

$-\cdots-E_{c}=0.9$

Figure 6: Effect of $E_{c}$ on velocity profiles.

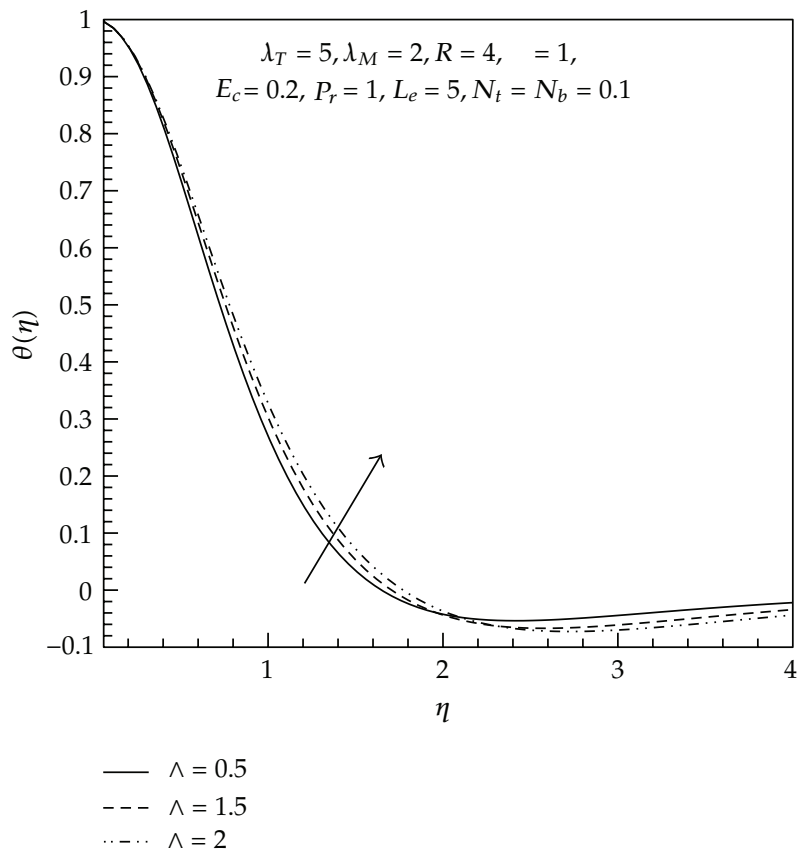

Figure 7: Effect of $\wedge$ on temperature profiles. 


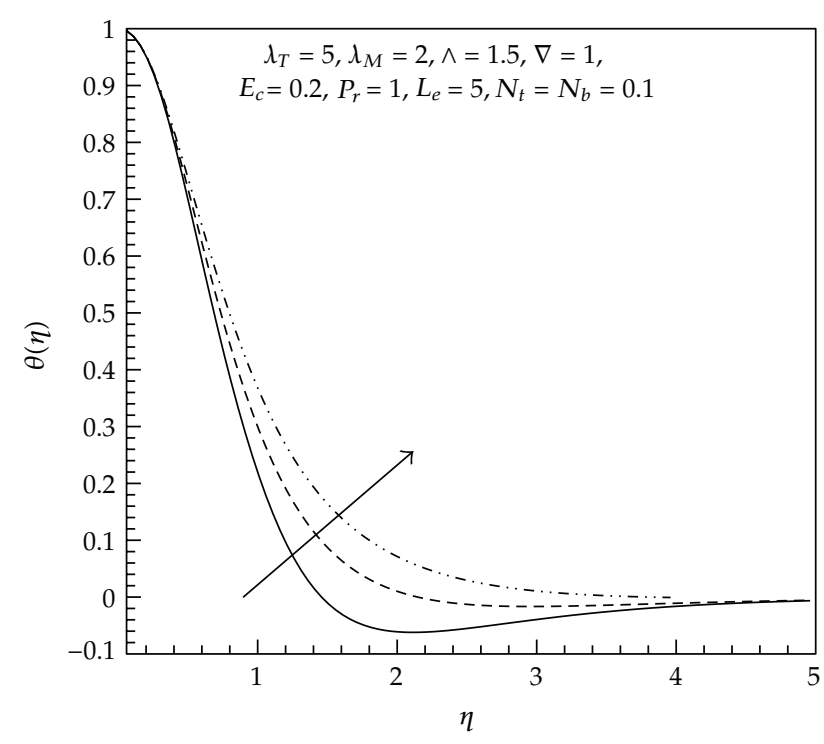

- $R=0.5$

- - $R=2.5$

-.- $R=5$

Figure 8: Effect of $R$ on temperature profiles.

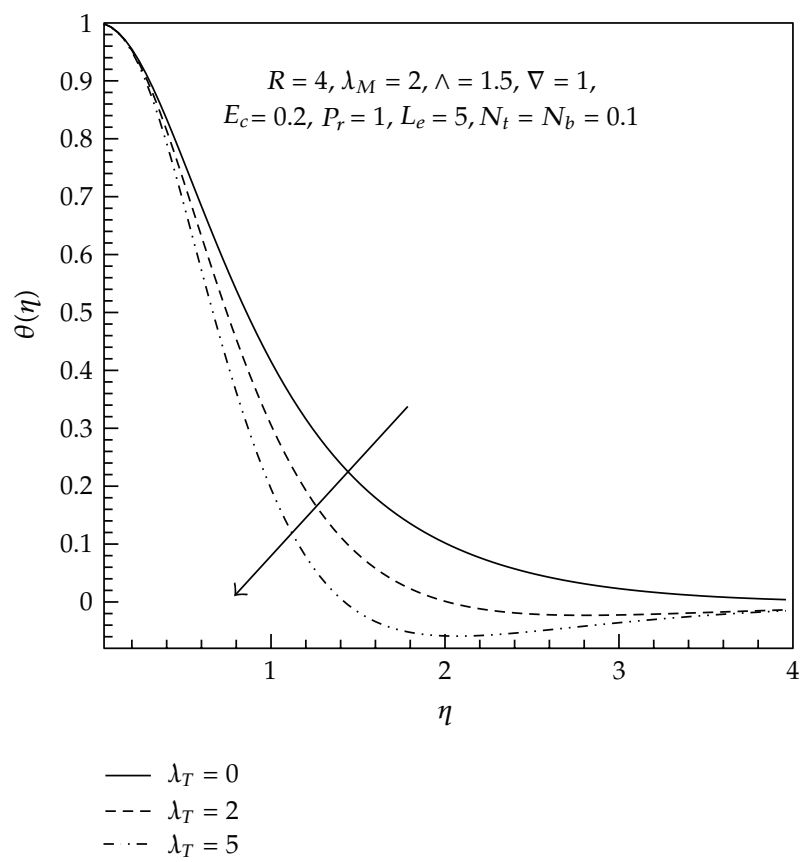

Figure 9: Effect of $\lambda_{T}$ on temperature profiles. 


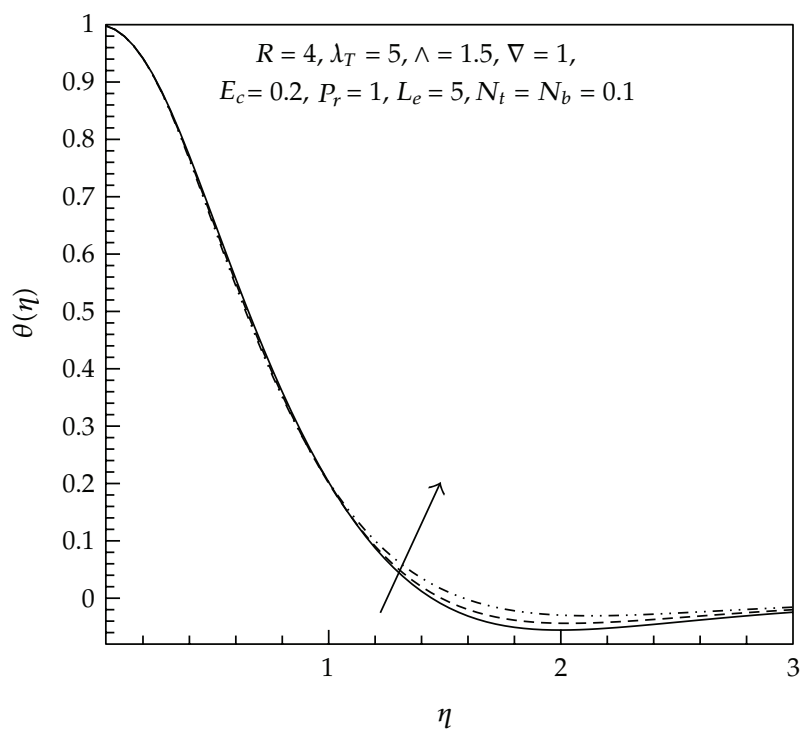

$-\lambda_{M}=0.5$

$--\lambda_{M}=1.5$

$-\cdots-\lambda_{M}=2.5$

Figure 10: Effect of $\lambda_{M}$ on temperature profiles.

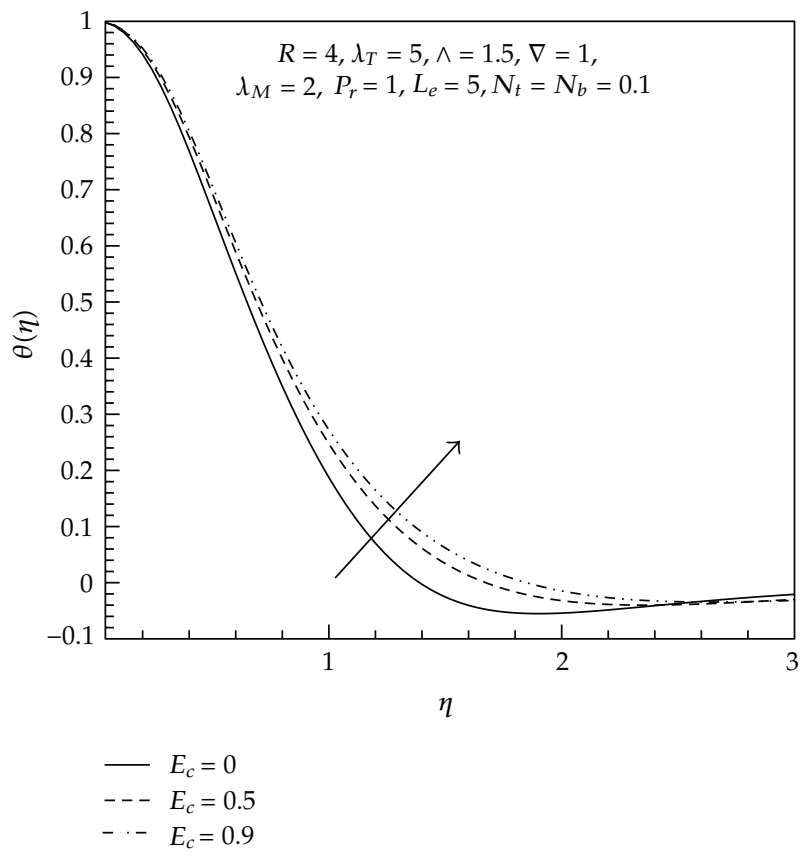

Figure 11: Effect of $E_{c}$ on temperature profiles. 


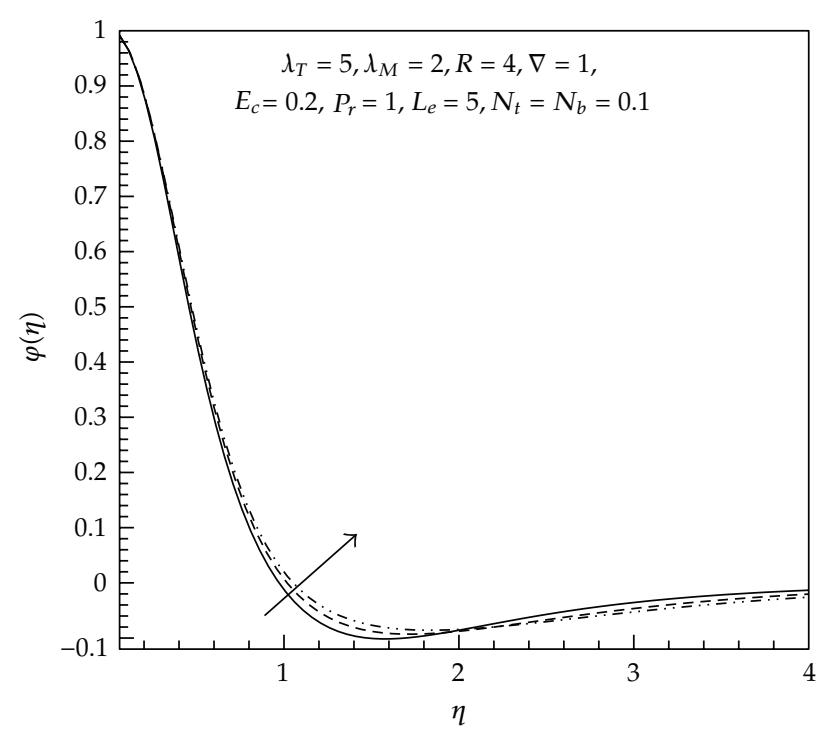

$-\wedge=0.5$

$--\wedge=1.5$

$-\cdots-\wedge=2$

Figure 12: Effect of $\wedge$ on concentration profiles.

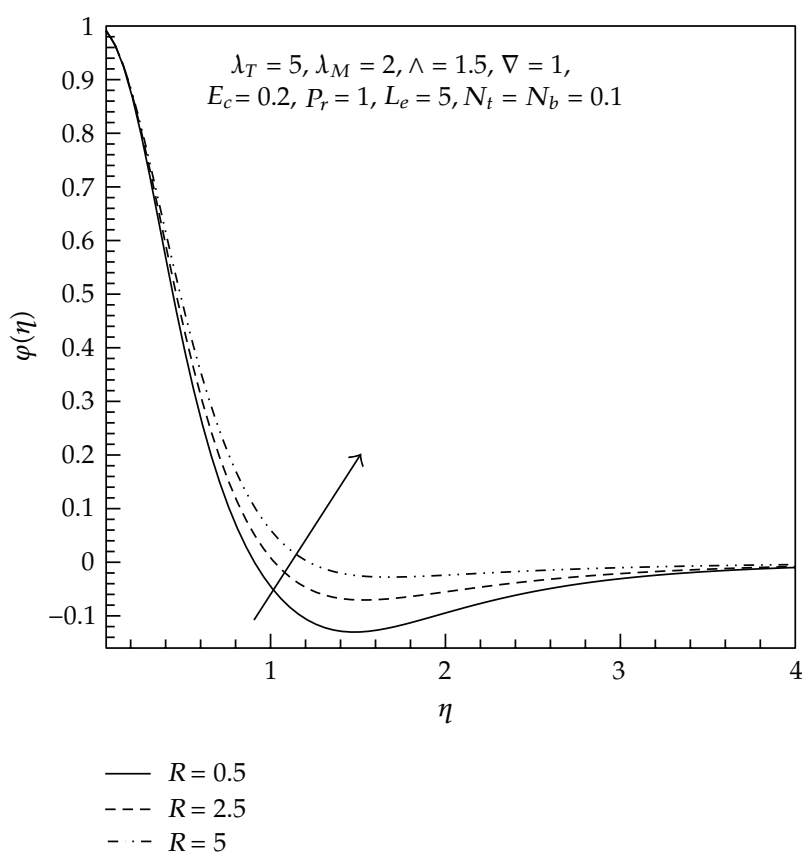

Figure 13: Effect of $R$ on concentration profiles. 


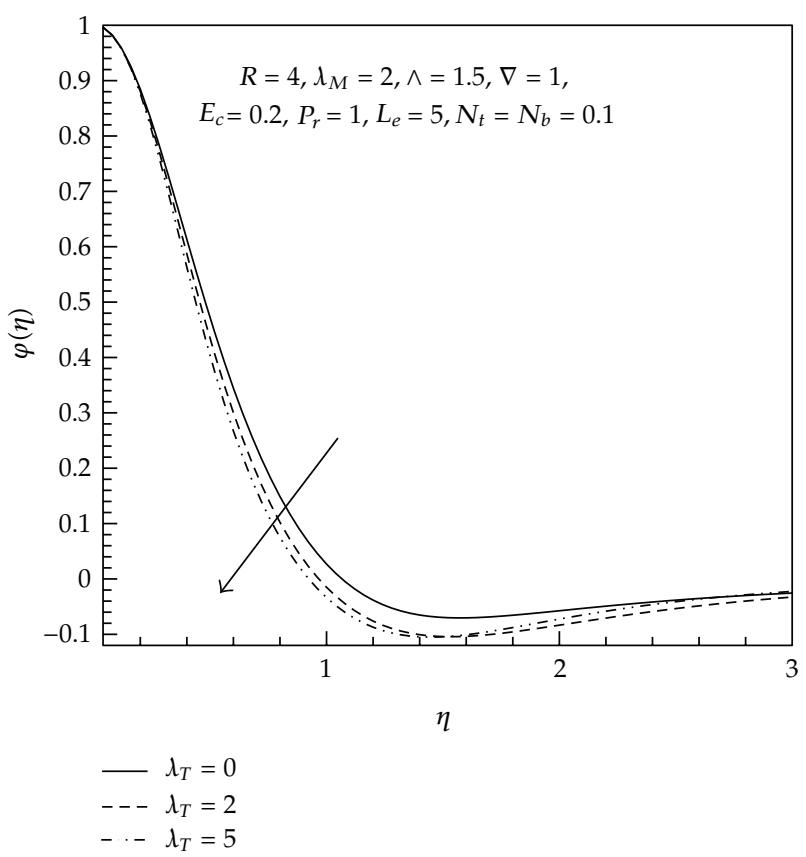

Figure 14: Effect of $\lambda_{T}$ on concentration profiles.

Figure 14 depicts the dimensionless concentration distribution $\varphi(\eta)$ for different values of $\lambda_{T}$ where, $\wedge=1.5, \lambda_{M}=2.0, R=4.0, \nabla=1.0, E_{c}=0.2, P_{r}=1.0, L_{e}=5.0, N_{t}=$ 0.1 , and $N_{b}=0.1$. It is observed that the increasing effect of $\lambda_{T}$ is to reduce concentration distribution as concentration species is dispersed away.

Figure 15 exhibits the dimensionless concentration distribution $\varphi(\eta)$ for different values of $\lambda_{M}$ where $\lambda_{T}=5.0, \wedge=1.5, R=4.0, \nabla=1.0, E_{c}=0.2, P_{r}=1.0, L_{e}=5.0, N_{t}=$ 0.1 , and $N_{b}=0.1$. It shows that the concentration boundary layer reduces as $\lambda_{M}$ is increased.

Figure 16 shows the dimensionless concentration distribution $\varphi(\eta)$ for different values of $E_{c}$ where $\lambda_{T}=5.0, \lambda_{M}=2.0, R=4.0, \nabla=1.0, \wedge=1.5, P_{r}=1.0, L_{e}=5.0, N_{t}=$ 0.1 , and $N_{b}=0.1$. It shows that the concentration boundary layer decreases gradually as $E_{c}$ is increased.

Since the physical interest of the problem, the skin-friction coefficient $\left(-f^{\prime \prime}\right)$, the Nusselt number $\left(-\theta^{\prime}\right)$ at the sheet and the Sherwood number $\left(-\varphi^{\prime}\right)$ at the sheet are plotted against Brownian motion parameter $\left(N_{b}\right)$ and illustrated in Figures 17-21.

Figure 17 illustrates the skin-friction coefficient $\left(-f^{\prime \prime}\right)$ plotted for the different values of $\wedge$ where $\lambda_{T}=5.0, \lambda_{M}=2.0, R=4.0, \nabla=1.0, E_{c}=0.2, P_{r}=1.0, L_{e}=5.0$, and $N_{b}=0.1$. It is verified that there is a decrease in skin-friction coefficient as $\wedge$ increases.

Figure 18 displays the skin-friction coefficient $\left(-f^{\prime \prime}\right)$ plotted for the different values of $R$ where $\lambda_{T}=5.0, \lambda_{M}=2.0, \wedge=1.5, \nabla=1.0, E_{c}=0.2, P_{r}=1.0, L_{e}=5.0$, and $N_{b}=0.1$. It is verified that there is a decrease in skin-friction coefficient as $R$ increases.

Figure 19 depicts the Nusselt number $\left(-\theta^{\prime}\right)$ plotted for the different values of $\wedge$ where $\lambda_{T}=5.0, \lambda_{M}=2.0, R=4.0, \nabla=1.0, E_{c}=0.2, P_{r}=1.0, L_{e}=5.0$, and $N_{b}=0.1$. It is verified that there is decrease in Nusselt number as $\wedge$ increases.

Figure 20 displays the Nusselt number $\left(-\theta^{\prime}\right)$ plotted for the different values of $R$ where $\lambda_{T}=5.0, \lambda_{M}=2.0, \wedge=1.5, \nabla=1.0, E_{c}=0.2, P_{r}=1.0, L_{e}=5.0$, and $N_{b}=0.1$. It is verified that there is a decrease in Nusselt number as $R$ increases. 


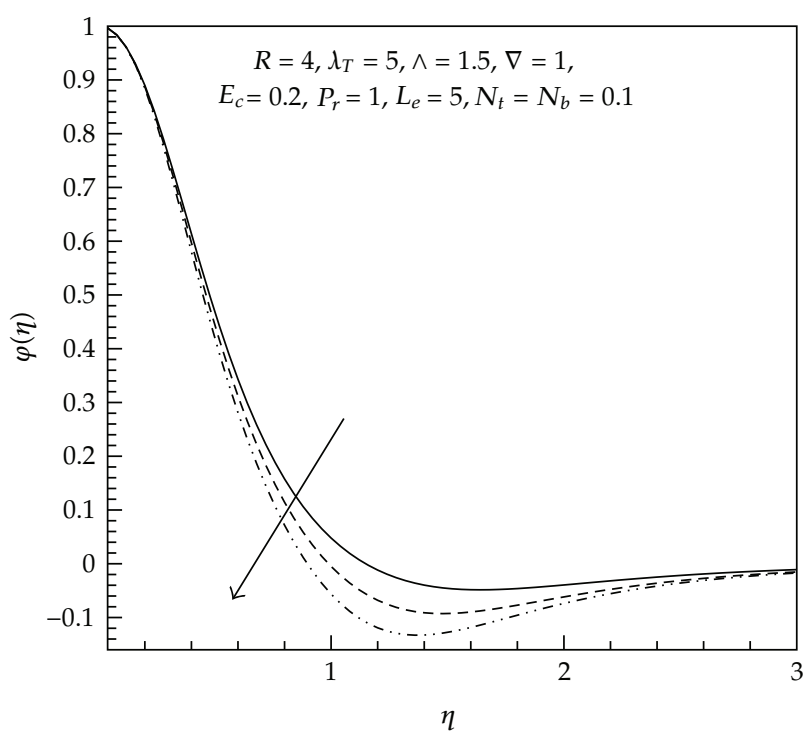

$-\lambda_{M}=0.5$

$--\lambda_{M}=1.5$

$-\cdots-\lambda_{M}=2.5$

Figure 15: Effect of $\lambda_{M}$ on concentration profiles.

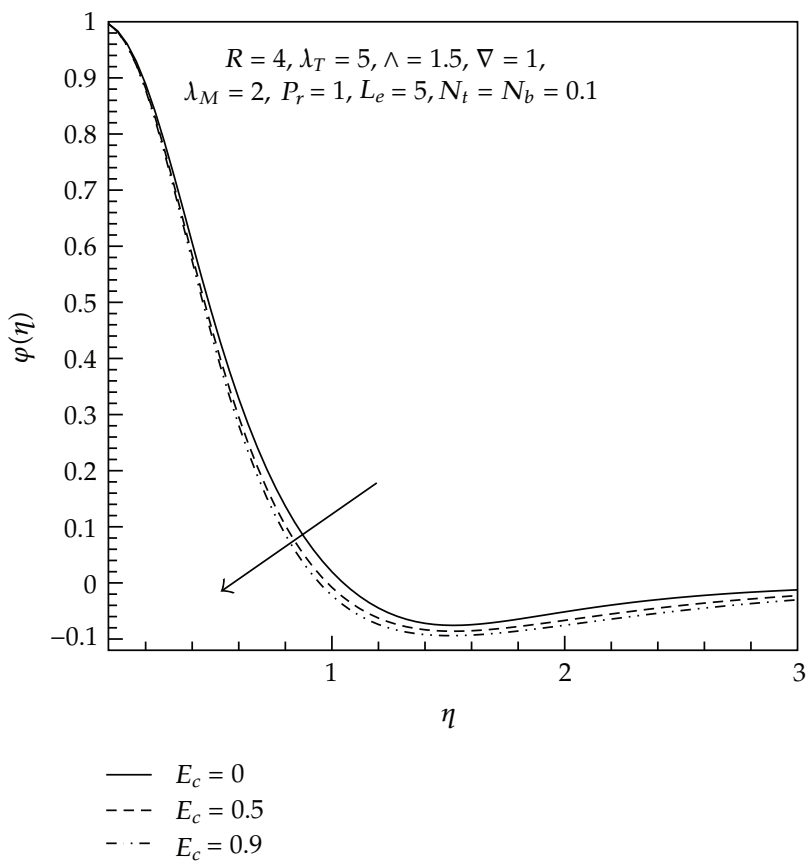

Figure 16: Effect of $E_{c}$ on concentration profiles. 


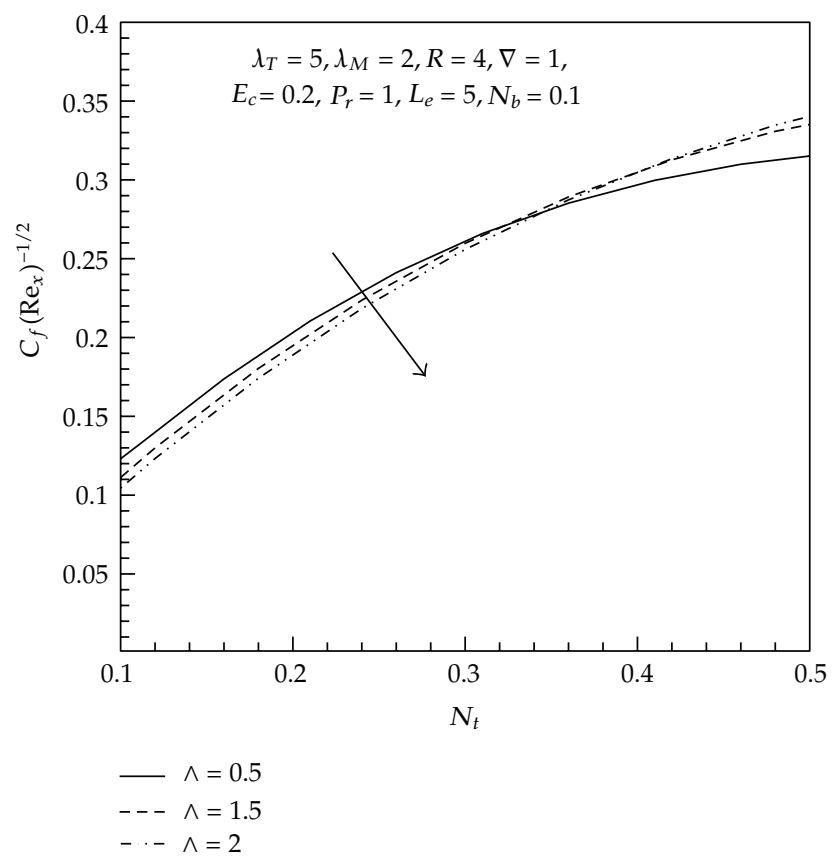

Figure 17: Effect of $\wedge$ on Skin-friction coefficient.

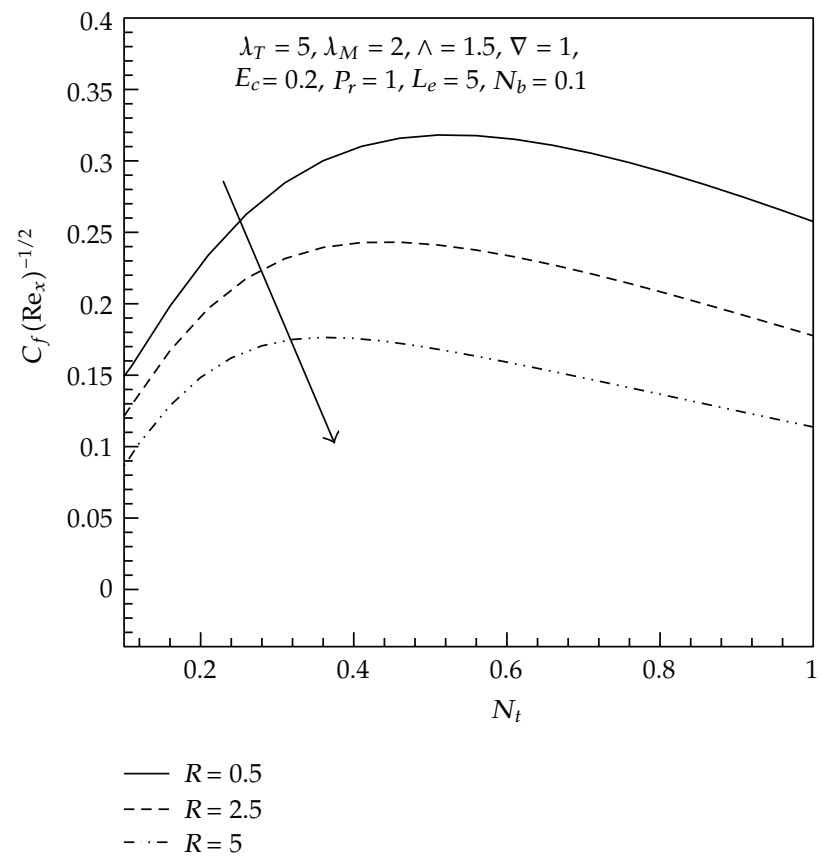

Figure 18: Effect of $R$ on skin-friction coefficient. 


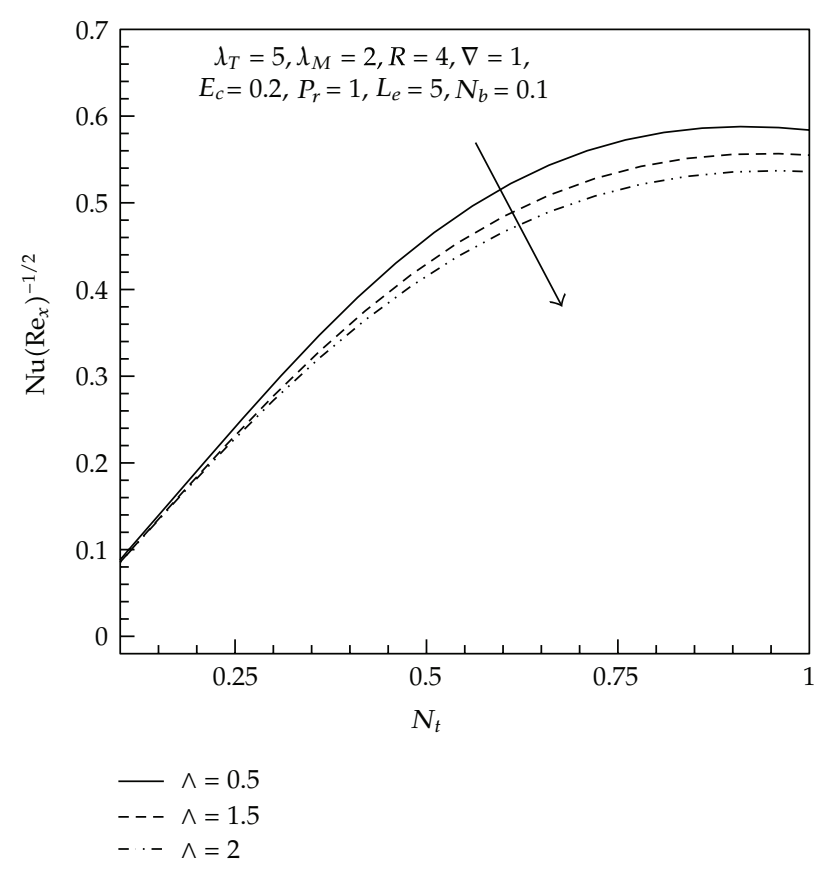

Figure 19: Effect of $\wedge$ on heat transfer rate.

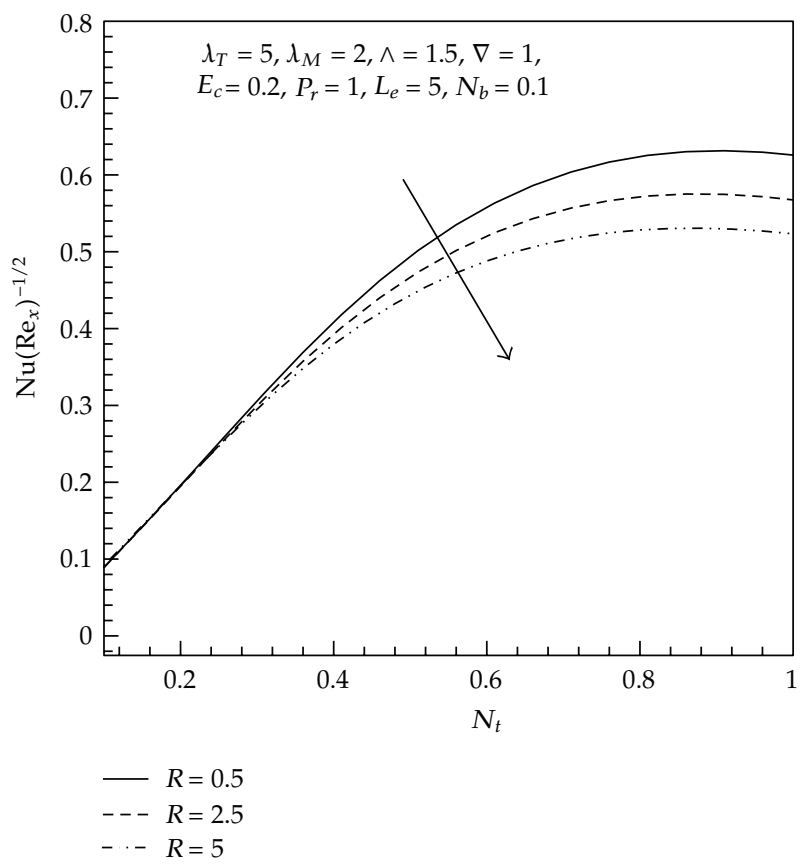

Figure 20: Effect of $R$ on heat transfer rate. 


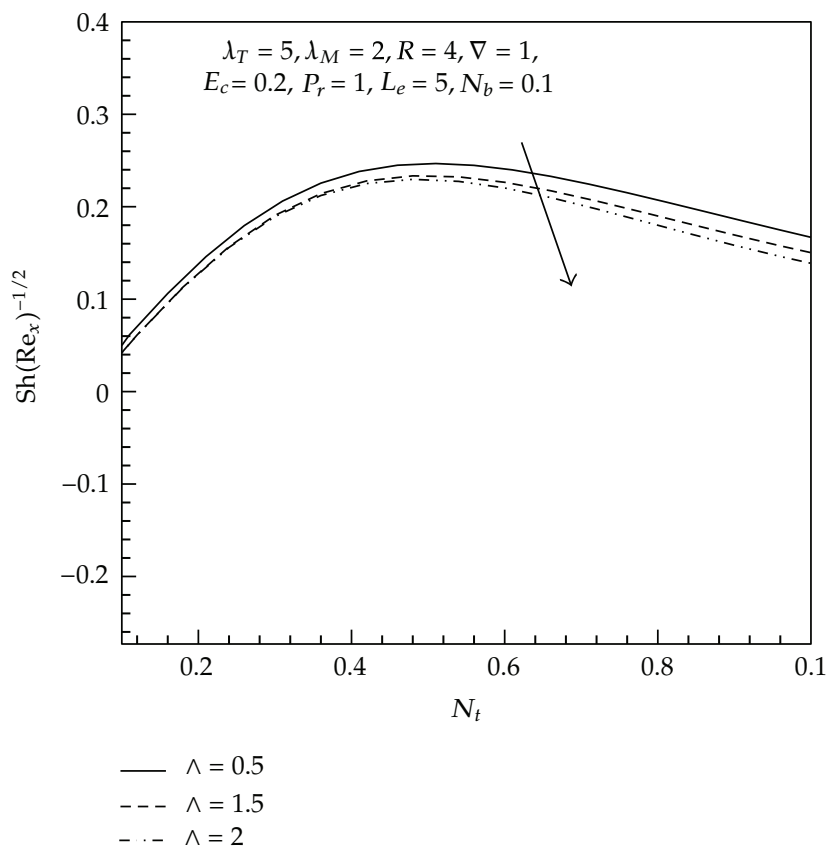

Figure 21: Effect of $\wedge$ on mass transfer rate.

Figure 21 shows the Sherwood number $\left(-\varphi^{\prime}\right)$ plotted for the different values of $\wedge$ where $\lambda_{1}=4.0, \alpha=1.0, F_{w}=2.0, Q=1.0, N_{t}=0.5, M=1.0, P_{r}=0.71$, and $L_{e}=5.0$. It is verified that there is a decrease in Sherwood number as $\wedge$ increases.

\section{Conclusions}

The effect of radiation on steady MHD mixed convection boundary layer flow of a nanofluid over an exponentially stretching sheet was investigated. The results are presented for the effect of various parameters. The velocity, temperature, and concentration effects as well as the skin-friction coefficient, Nusselt number, and Sherwood number effects along the exponential sheet are studied and shown graphically. However, the comparison with previously published work [11] is performed and excellent agreement is observed. From the present study the important findings are listed below.

(1) Momentum, thermal, and concentration boundary layer thickness increase as the viscous ratio parameter increases. Whereas skin-friction coefficient, surface heat, and mass transfer rate decreases.

(2) Momentum boundary layer thickness reduces; on the other hand, thermal and concentration boundary layer increases steeply near the exponential stretching sheet as the combined porous and Magnetic parameter is increased. Whereas skinfriction coefficient and surface heat transfer rate decreases.

(3) Momentum and thermal boundary layer increase whereas the concentration boundary layer decreases gradually as the Eckert number is increased. 


\section{Nomenclature}

$B_{0}$ : Magnetic induction

C: Nanoparticle concentration

$C_{0}$ : Reference concentration

$C_{w}$ : Nanoparticle concentration at stretching surface

$C_{\infty}$ : Ambient nanoparticle concentration as $y$ tends to infinity

$C_{f}$ : Skin-friction coefficient

$c_{p}$ : Specific heat capacity

$D_{B}$ : Brownian diffusion coefficient

$D_{T}$ : Thermophoresis diffusion coefficient

g: Acceleration due to gravity

$G_{r}$ : Grashof number

$G_{m}$ : Modified Grashof number

$L_{e}$ : Lewis number

$L: \quad$ Reference length

$N_{u}$ : Nusselt number

$N_{b}$ : Brownian motion parameter

$N_{t}$ : Thermophoresis parameter

$P$ : $\quad$ Fluid pressure

$P_{r}$ : Prandtl number

$R$ : Combined porous and magnetic parameter

$R_{e}$ : Local Reynolds number

$S_{h}$ : Sherwood number

$T$ : Fluid temperature

$T_{\circ}$ : Reference temperature

$T_{w}$ : Temperature at the stretching surface

$T_{\infty}$ : Ambient temperature as $y$ tends to infinity

$u, v$ : Velocity components along $x$-and $y$-axes, respectively

$u_{\circ}$ : Reference velocity

$x, y$ : Cartesian coordinates measured along stretching surface.

\section{Greek Symbols}

v: $\quad$ Kinematic viscosities

$\tilde{v}$ : $\quad$ Reference kinematic viscosity

$(\rho c)_{p}$ : Effective heat capacity of the nanofluid

$(\rho c)_{f}$ : Heat capacity of the fluid

$\alpha$ : $\quad$ Thermal diffusivity

$\beta$ : $\quad$ Coefficient of thermal expansion

$\lambda_{T}$ : Thermal convective parameter

$\lambda_{M}$ : Mass convective parameter

$\eta$ : $\quad$ Similarity variable

$\psi: \quad$ Stream function

$f^{\prime}(\eta)$ : Dimensionless velocity

$\theta(\eta)$ : Dimensionless temperature

$\varphi(\eta)$ : Dimensionless concentration. 


\section{References}

[1] L. J. Crane, "Flow past a stretching plate," Journal of Applied Mathematics and Physics, vol. 21, no. 4, pp. 645-647, 1970.

[2] P. Carragher and L. Crane, "Heat transfer on continuous stretching sheet," Journal of Applied Mathematics and Mechanics, vol. 10, no. 62, pp. 564-565, 1982.

[3] E. Magyari and B. Keller, "Heat and mass transfer in the boundary layers on an exponentially stretching continuous surface," Journal of Physics D, vol. 32, no. 5, pp. 577-585, 1999.

[4] M. K. Partha, P. V. S. N. Murthy, and G. P. Rajasekhar, "Effect of viscous dissipation on the mixed convection heat transfer from an exponentially stretching surface," Heat and Mass Transfer/Waermeund Stoffuebertragung, vol. 41, no. 4, pp. 360-366, 2005.

[5] M. Sajid and T. Hayat, "Influence of thermal radiation on the boundary layer flow due to an exponentially stretching sheet," International Communications in Heat and Mass Transfer, vol. 35, no. 3, pp. 347-356, 2008.

[6] P. Ganesan and G. Palani, "Finite difference analysis of unsteady natural convection MHD flow past an inclined plate with variable surface heat and mass flux," International Journal of Heat and Mass Transfer, vol. 47, no. 19-20, pp. 4449-4457, 2004.

[7] M. A. Seddeek, "Effects of radiation and variable viscosity on a MHD free convection flow past a semiinfinite flat plate with an aligned magnetic field in the case of unsteady flow," International Journal of Heat and Mass Transfer, vol. 45, no. 4, pp. 931-935, 2001.

[8] E. M. A. Elbashbeshy, "Heat transfer over an exponentially stretching continuous surface with suction," Archives of Mechanics, vol. 53, no. 6, pp. 643-651, 2001.

[9] S. K. Khan, "Boundary layer viscoelastic fluid flow over an exponentially stretching sheet," International Journal of Applied Mechanics and Engineering, vol. 11, pp. 321-335, 2006.

[10] E. Sanjayanand and S. K. Khan, "On heat and mass transfer in a viscoelastic boundary layer flow over an exponentially stretching sheet," International Journal of Thermal Sciences, vol. 45, no. 8, pp. 819-828, 2006.

[11] B. Bidin and R. Nazar, "Numerical solution of the boundary layer flow over an exponentially stretching sheet with thermal radiation," European Journal of Scientific Research, vol. 33, no. 4, pp. 710$717,2009$.

[12] C. Y. Wang, "Free convection on a vertical stretching surface," Journal of Applied Mathematics and Mechanics, vol. 69, pp. 418-420, 1989.

[13] R. S. Reddy Gorla and I. Sidawi, "Free convection on a vertical stretching surface with suction and blowing," Applied Scientific Research, vol. 52, no. 3, pp. 247-257, 1994.

[14] K. Vajravelu and A. Hadjinicolaou, "Heat transfer in a viscous fluid over a stretching sheet with viscous dissipation and internal heat generation," International Communications in Heat and Mass Transfer, vol. 20, no. 3, pp. 417-430, 1993.

[15] P. Cheng and W. J. Minkowycz, "Free convection about a vertical flat plate embedded in a porous medium with application to heat transfer from a dike," Journal of Geophysical Research, vol. 82, no. 14, pp. 2040-2044, 1977.

[16] R. S. R. Gorla and A. H. Zinalabedini, "Free convection from a vertical plate with nonuniform surface temperature and embedded in a porous medium," Journal of Energy Resources Technology, vol. 109, no. 1, pp. 26-30, 1987.

[17] R. S. R. Gorla and R. Tornabene, "Free convection from a vertical plate with nonuniform surface heat flux and embedded in a porous medium," Transport in Porous Media Journal, vol. 3, no. 1, pp. 95-106, 1988.

[18] S. U. S. Choi, "Enhancing thermal conductivity of fluids with nanoparticles," in Development and Applications of Non-Newtonian Flows, D. A. Siginer and H. P. Wang, Eds., pp. 99-105, Washington, DC, USA, 1995, ASME MD- vol. 231 and FED-vol. 66, USDOE.

[19] H. U. Kang, S. H. Kim, and J. M. Oh, "Estimation of thermal conductivity of nanofluid using experimental effective particle volume," Experimental Heat Transfer, vol. 19, no. 3, pp. 181-191, 2006.

[20] E. Abu-Nada, "Application of nanofluids for heat transfer enhancement of separated flows encountered in a backward facing step," International Journal of Heat and Fluid Flow, vol. 29, no. 1, pp. 242-249, 2008.

[21] E. Abu-Nada and H. F. Oztop, "Effects of inclination angle on natural convection in enclosures filled with Cu-water nanofluid," International Journal of Heat and Fluid Flow, vol. 30, no. 4, pp. 669-678, 2009.

[22] X.-Q. Wang and A. S. Mujumdar, "Heat transfer characteristics of nanofluids: a review," International Journal of Thermal Sciences, vol. 46, pp. 1-19, 2007. 
[23] X.-Q Wang and A. S. Mujumdar, "A review on nanofluids-Part I: theoretical and numerical investigations," Brazilian Journal of Chemical Engineering, vol. 25, pp. 613-630, 2008.

[24] B. Ghasemi and S. M. Aminossa dati, "Periodic natural convection in a nanofluid filled enclosure with oscillating heat flux," International Journal of Thermal Sciences, vol. 49, pp. 1-9, 2010.

[25] J. Kim, Y. T. Kang, and C. K. Choi, "Analysis of convective instability and heat transfer characteristics of nanofluids," Physics of Fluids, vol. 16, no. 7, pp. 2395-2401, 2004.

[26] S. P. Jang and S. U.S. Choi, "Effects of various parameters on nanofluid thermal conductivity," Journal of Heat Transfer, vol. 129, no. 5, pp. 617-623, 2007.

[27] D. A. Nield and A. V. Kuznetsov, "The Cheng-Minkowycz problem for natural convective boundarylayer flow in a porous medium saturated by a nanofluid," International Journal of Heat and Mass Transfer, vol. 52, no. 25-26, pp. 5792-5795, 2009.

[28] A. V. Kuznetsov and D. A. Nield, "Natural convective boundary-layer flow of a nanofluid past a vertical plate," International Journal of Thermal Sciences, vol. 49, no. 2, pp. 243-247, 2010.

[29] A. S. Monin and A. M. Yaglom, Statistical Fluid Mechanics: Mechanics of Turbulence, vol. 2, The MIT Press, Cambridge, Mass, USA, 1971.

[30] S. C. Lim and S. V. Muniandy, "On some possible generalizations of fractional Brownian motion," Physics Letters A, vol. 266, no. 2-3, pp. 140-145, 2000.

[31] S. C. Lim, "Fractional Brownian motion and multifractional Brownian motion of Riemann-Liouville type," Journal of Physics A, vol. 34, no. 7, pp. 1301-1310, 2001.

[32] M. Li and W. Zhao, "Quantitatively investigating the locally weak stationarity of modified multifractional Gaussian noise," Physica A, vol. 391, no. 24, pp. 6268-6278, 2012.

[33] M. Li, W. Zhao, and S. Chen, "MBm-based scalings of traffic propagated in internet," Mathematical Problems in Engineering, vol. 2011, Article ID 389803, 21 pages, 2011.

[34] M. Li, "Fractal time series-a tutorial review," Mathematical Problems in Engineering, vol. 2010, Article ID 157264, 26 pages, 2010.

[35] R. F. Peltier and J. Levy-Vehel, "A new method for estimating the parameter of fractional brownian motion," INRIA RR 2696, 1994.

[36] J.-P. Bouchaud and A. Georges, "Anomalous diffusion in disordered media: statistical mechanisms, models and physical applications," Physics Reports A, vol. 195, no. 4-5, pp. 127-293, 1990.

[37] N. Bachok, A. Ishak, and I. Pop, "Boundary-layer flow of nanofluids over a moving surface in a flowing fluid," International Journal of Thermal Sciences, vol. 49, no. 9, pp. 1663-1668, 2010.

[38] W. A. Khan and I. Pop, "Boundary-layer flow of a nanofluid past a stretching sheet," International Journal of Heat and Mass Transfer, vol. 53, no. 11-12, pp. 2477-2483, 2010.

[39] W. A. Khan and I. Pop, "Free convection boundary layer flow past a horizontal flat plate embedded in a porous medium filled with a nanofluid," Journal of Heat Transfer, vol. 133, no. 9, Article ID 094501, 2011.

[40] M. A. A. Hamad and I. Pop, "Scaling transformations for boundary layer flow near the stagnationpoint on a heated permeable stretching surface in a porous medium saturated with a nanofluid and heat generation/absorption effects," Transport in Porous Media, vol. 87, no. 1, pp. 25-39, 2011.

[41] M. A. A. Hamad, I. Pop, and A. I. M. Ismail, "Magnetic field effects on free convection flow of a nanofluid past a vertical semi-infinite flat plate," Nonlinear Analysis: Real World Applications, vol. 12, no. 3, pp. 1338-1346, 2011.

[42] M. Shakhaoath Khan, M. Mahmud Alam, and M. Ferdows, "Finite difference solution of MHD radiative boundary layer flow of a nanofluid past a stretching sheet," in Proceeding of the International Conference of Mechanical Engineering (ICME '11), BUET, Dhaka, Bangladesh, 2011, FL-011.

[43] M. Shakhaoath Khan, M. Mahmud Alam, and M. Ferdows, "MHD radiative boundary layer flow of a nanofluid past a stretching sheet," in Proceeding of the International Conference of Mechanical Engineering and Renewable Energy (ICMERE '11), CUET, Chittagong, Bangladesh, 2011, PI-105.

[44] P. R. Nachtsheim and P. Swigert, "Satisfaction of the asymptotic boundary conditions in numerical solution of the system of non-linear equations of boundary layer type," NASA TND-3004, 1965. 


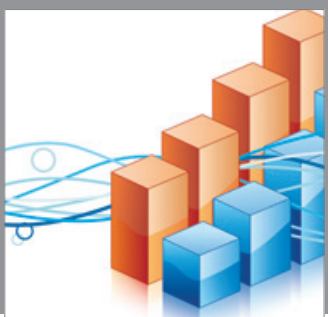

Advances in

Operations Research

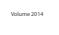

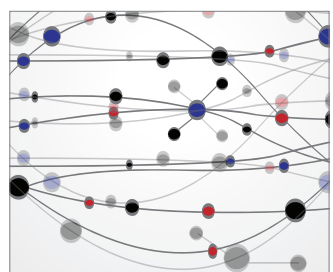

\section{The Scientific} World Journal
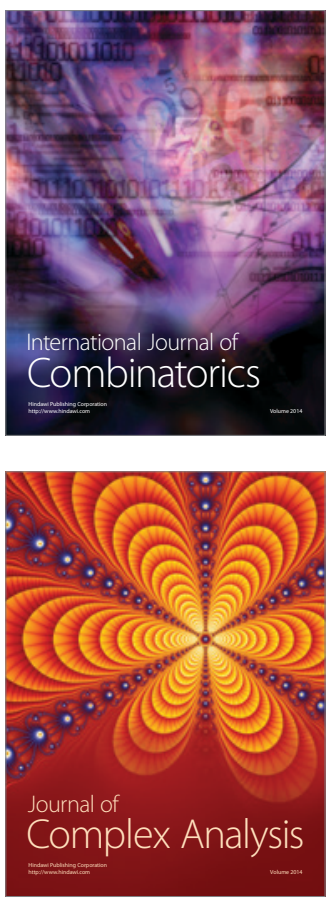

International Journal of

Mathematics and

Mathematical

Sciences
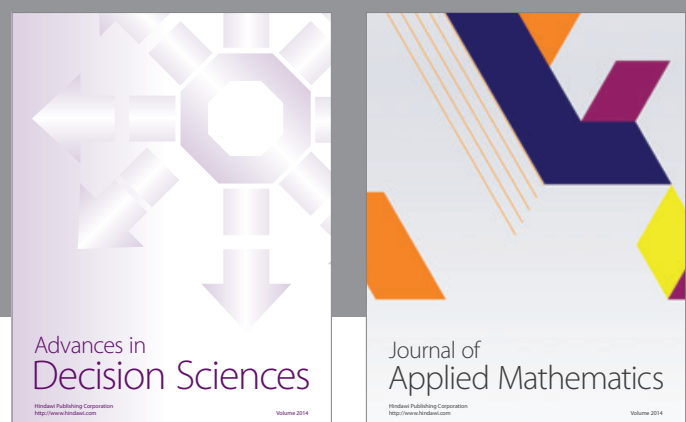

Journal of

Applied Mathematics
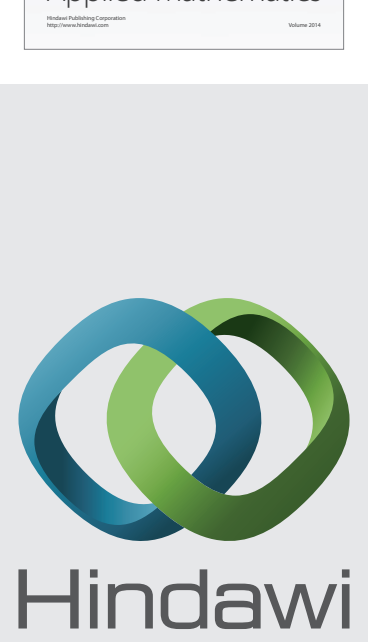

Submit your manuscripts at http://www.hindawi.com
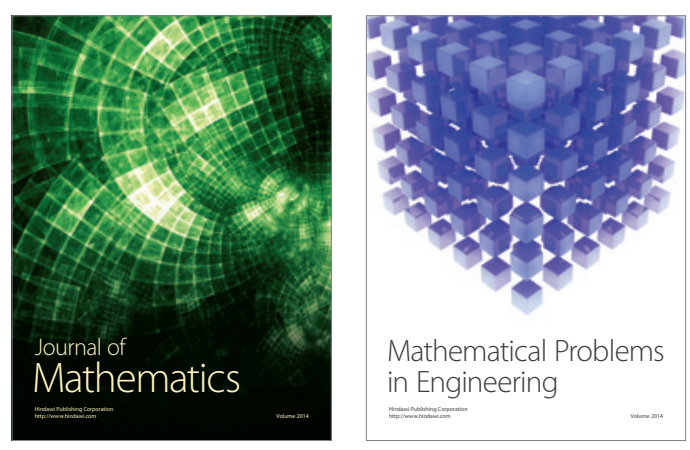

Mathematical Problems in Engineering
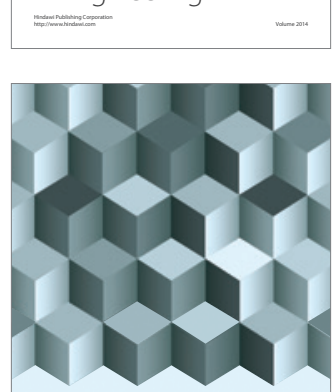

Journal of

Function Spaces
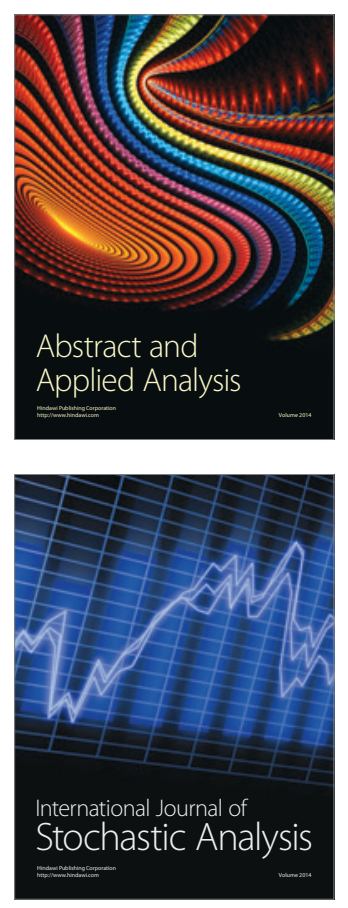

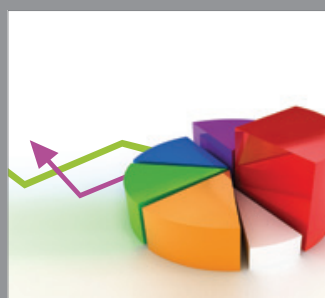

ournal of

Probability and Statistics

Promensencen
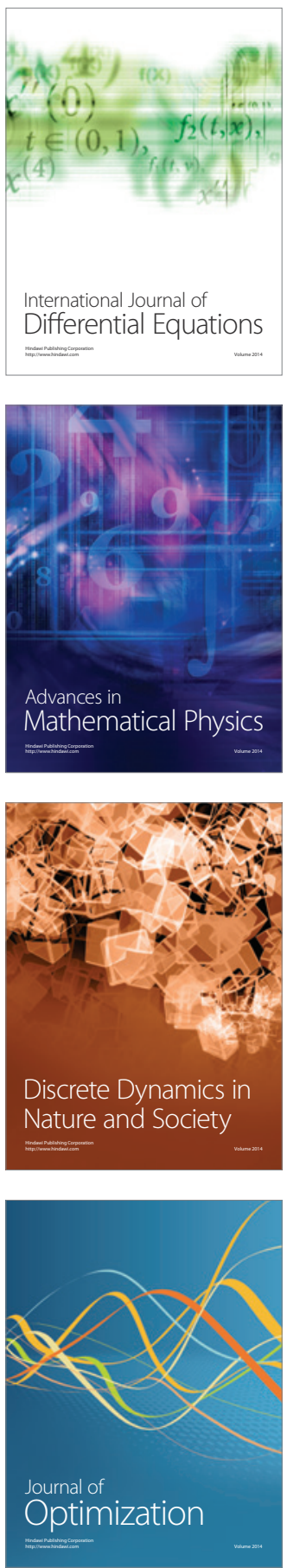